Mathematical Modelling and AnAlysis

Volume 16 Number 3, September 2011, 461-474

Doi:10.3846/13926292.2011.603164

(c) Vilnius Gediminas Technical University, 2011
Publisher: Taylor\&Francis and VGTU

www.tandfonline.com/loi/tmma20

Online ISSN: $1648-3510$

Print ISSN: 1392-6292

\title{
Stationary Patterns of a Ratio-Dependent Predator-Prey System with Cross-Diffusion*
}

\author{
Yu-Xia Wang, Wan-Tong Li and Hong-Bo Shi \\ School of Mathematics and Statistics, Lanzhou University \\ Lanzhou, Gansu 730000, China \\ E-mail: yxwang_10@lzu.edu.cn; shihb07@lzu.cn \\ E-mail(corresp.): wtli@lzu.edu.cn
}

Received November 10, 2009; revised June 28, 2011; published online August 1, 2011

\begin{abstract}
This paper is concerned with a ratio-dependent predator-prey system with diffusion and cross-diffusion in a bounded domain with no flux boundary condition. We establish the existence and non-existence of non-constant positive steady states (patterns). In particular, we show that under certain hypotheses, the crossdiffusion can create stationary patterns even though the corresponding model without cross-diffusion fails.
\end{abstract}

Keywords: existence, predator-prey system, cross-diffusion, positive steady state, ratiodependence.

AMS Subject Classification: 35K57; 35R20; 92D25.

\section{Introduction}

Interaction between the predator and prey influences the population growth of both species, which has been observed from the population data of Canadian lynx and snowshoe hare since the 1840s. Moreover, predator-prey systems can generate rich dynamics and have been of great interest to both applied mathematicians and ecologists since last century. In view that the classical Lotka-Volterra models have unavoidable limitation to precisely describe many realistic phenomena in biological systems in some cases, a major trend in theoretical work on predator-prey dynamics has been launched so as to derive more realistic models and functional responses. Recently, many authors considered

\footnotetext{
* Supported by NSFC (No.10871085).
} 
the following predator-prey system:

$$
\left\{\begin{array}{l}
u_{t}=d_{1} \Delta u+u g(u)-v p(u), \quad x \in \Omega, t>0, \\
v_{t}=d_{2} \Delta v+b v-\frac{v^{2}}{\gamma u}, \quad x \in \Omega, t>0, \\
\partial_{\nu} u=\partial_{\nu} v=0, \quad x \in \partial \Omega, t>0, \\
u(x, 0)=u_{0}(x), \quad v(x, 0)=v_{0}(x), \quad x \in \bar{\Omega},
\end{array}\right.
$$

where $\Omega \subseteq \mathbb{R}^{N}$ is a bounded domain with smooth boundary $\partial \Omega$; $u$ and $v$ represent the species densities of prey and predator, respectively; the parameter $b$ is the intrinsic growth rate of the predator; $\gamma$ takes on the role of the preydependent carrying capacity for the predator; the constants $d_{i}(i=1,2)$ are the diffusion coefficients for $u$ and $v$, respectively; $\nu$ is the outward unit normal vector on the boundary $\partial \Omega$ and $\partial_{\nu}=\partial / \partial \nu$. The homogeneous Neumann boundary condition means that (1.1) is self-contained, thus has no population flux across the boundary $\partial \Omega$. The initial data $u_{0}(x)$ and $v_{0}(x)$ are smooth non-negative functions on $\bar{\Omega}$. Biologically, all the parameters appearing in the system are assumed to be positive. A typical case of $g$ is the logistic type, namely, $g(u)=r(1-u / k) \cdot p(u)$ is called the functional response.

Recently, there are some papers concerned with the existence of non constant positive steady states of (1.1). In particular, Peng and Wang [22] obtained the existence and non-existence of positive non-constant steady states of (1.1) with $p(u)=q u /(u+m)$. Furthermore, Ko and Ryu [12] also considered the existence and non-existence of non-constant positive steady states of (1.1) with general functions $g(u)$ and $p(u)$.

There is growing biological and physical evidence that in many situations, such as when predators have to search, share and compete for food, a more suitable predator-prey model should be based on the ratio-dependent theory (see $[1,2])$. On the other hand, in population dynamics, one of the observed features is that different concentration levels of the prey direct the movements of the predator and vice versa. In other words, the movement of a predator at any particular location is influenced by the gradient of the concentration of the prey at that location, and the movement of the prey is affected by the gradient of the concentration of the predator at the same location. Taking this into account, we consider the following ratio-dependent predator-prey system with diffusion and cross-diffusion

$$
\left\{\begin{array}{l}
u_{t}-d_{1} \Delta u=r u\left(1-\frac{u}{k}\right)-\frac{\beta u v}{u+m v}, \quad x \in \Omega, t>0, \\
v_{t}-d_{2} \Delta\left[\left(1+d_{3} u\right) v\right]=b v-\frac{v^{2}}{\gamma u}, \quad x \in \Omega, t>0, \\
\partial_{\nu} u=\partial_{\nu} v=0, \quad x \in \partial \Omega, t>0, \\
u(x, 0)=u_{0}(x), \quad v(x, 0)=v_{0}(x), \quad x \in \bar{\Omega} .
\end{array}\right.
$$

Here, the parameter $m$ represents the saturation rate; in (1.2), $d_{3}$ called the cross-diffusion coefficient, is a non-negative constant. We rewrite

$$
d_{2} \Delta\left[\left(1+d_{3} u\right) v\right]=d_{2} \operatorname{div}\left(\left(1+d_{3} u\right) \nabla v+d_{3} v \nabla u\right)
$$


and regard $-d_{2}\left(1+d_{3} u\right) \nabla v-d_{2} d_{3} v \nabla u$ as the flux of the predator $v$. If $d_{3}>0$, the term $-d_{2} d_{3} v \nabla u$ of the flux of the predator is directed towards the decreasing population density of $u$. More details about the cross-diffusion can be found in $[4,13,16,17,21,24,27]$.

For simplicity, we nondimensionalize system (1.2) with the following scaling $u \rightarrow u / k, v \rightarrow v /(\gamma k), t \rightarrow r k t$, then system (1.2) takes the form

$$
\left\{\begin{array}{l}
u_{t}-\frac{d_{1}}{k r} \Delta u=\frac{u}{k}(1-u)-\frac{\beta \gamma u v}{k r(u+m \gamma v)}, \quad x \in \Omega, t>0, \\
v_{t}-\frac{d_{2}}{k r} \Delta\left[\left(1+d_{3} k u\right) v\right]=\frac{v}{k r}\left(b-\frac{v}{u}\right), \quad x \in \Omega, t>0, \\
\partial_{\nu} u=\partial_{\nu} v=0, \quad x \in \partial \Omega, t>0, \\
u(x, 0)=u_{0}^{\prime}(x), \quad v(x, 0)=v_{0}^{\prime}(x), \quad x \in \bar{\Omega} .
\end{array}\right.
$$

For this kind of the partial differential equation model, an important issue is to find the non-constant positive steady state, which is related to the non-constant positive solution of the corresponding elliptic equations. Thus, in our paper, we shall study the following system

$$
\left\{\begin{array}{l}
-d_{1} \Delta u=r u(1-u)-\frac{\beta u v}{u+m v}, \quad x \in \Omega, \\
-d_{2} \Delta\left[\left(1+d_{3} u\right) v\right]=v(1-v / u), \quad x \in \Omega, \\
\partial_{\nu} u=\partial_{\nu} v=0, \quad x \in \partial \Omega .
\end{array}\right.
$$

Here, for simplicity, we take $b=1$ in system (1.3).

In the past decades, there have been many works on the existence and non-existence of non-constant positive steady states of ecological models with diffusion or cross-diffusion under the homogeneous Neumann boundary conditions. One can refer to $[4,3,11,12,19,20,21,22,24,26,27]$. The role of diffusion in modelling many physical, chemical and biological processes has been extensively studied. Starting with Turing's seminal paper [25], diffusion and cross-diffusion have been observed as causes of the spontaneous emergence of ordered structures, called patterns in a variety of non-equilibrium situations. They include the Gierer-Meinhardt model [7, 8, 28], the Sel'kov model $[5,26]$, the Lotka-Volterra competition model $[15,16,17]$ and the LotkaVolterra predator-prey model $[4,6,9,10,19,20]$ and so on.

The main purpose of this paper is to study the effect of the cross-diffusion pressure on the existence of non-constant positive steady states of (1.3). We will show that even though the unique constant solution is globally asymptotically stable for (1.3) with $d_{3}=0$, non-constant positive solution can still exist due to the emergence of the cross-diffusion. Our result implies that cross-diffusion can create stationary patterns. For the related results, the interested readers can refer to $[4,16,24]$ and references therein.

Throughout the paper, the positive solution of (1.3) refers to a classical one with $u>0, v>0$ on $\bar{\Omega}$. It is easy to see that (1.3) has a semi-trivial non-negative solution $\boldsymbol{u}^{0}=(1,0)$ and a unique positive constant solution $\boldsymbol{u}^{*}=$ $\left(u^{*}, v^{*}\right)$, where

$$
u^{*}=1-\frac{\beta}{r(1+m)}, \quad v^{*}=u^{*} \text {. }
$$


Obviously, $\boldsymbol{u}^{*}=\left(u^{*}, v^{*}\right)$ exits if and only if $\beta<r(1+m)$. In the sequel, we always assume $\beta<r(1+m)$, unless otherwise stated.

For non-constant solutions, there are two major methods in establishing the existence of nontrivial solutions to elliptic systems, namely, singular perturbation (see $[9,10]$ ) and bifurcation. A variation of the bifurcation technique is the powerful Leray-Schauder degree approach (see $[6,19,20,26])$ which will be used in our paper.

The organization of this paper is as follows. In Section 2, we first establish a priori upper and lower bounds for the positive solutions of (1.3). Section 3 deals with the non-existence of the non-constant positive solutions of (1.3). Finally, in Section 4, we establish the existence of non-constant positive solutions of (1.3) for a range of diffusion and cross-diffusion coefficients.

\section{A Priori Estimates for Positive Solutions}

In this section, we shall give a priori estimates for the positive solutions of (1.3). First, we recall the following two lemmas, which can be found in $[14,16]$.

Lemma 1 [Maximum Principle [16]]. Suppose that $g \in C(\bar{\Omega} \times \mathbb{R})$.

(i) Assume that $w \in C^{2}(\Omega) \cap C^{1}(\bar{\Omega})$ and satisfies

$$
\begin{gathered}
\Delta w(x)+g(x, w(x)) \geqslant 0 \quad \text { in } \Omega, \partial_{\nu} w \leqslant 0 \quad \text { on } \partial \Omega . \\
\text { If } w\left(x_{0}\right)=\max _{\bar{\Omega}} w \text {, then } g\left(x_{0}, w\left(x_{0}\right)\right) \geqslant 0 .
\end{gathered}
$$

(ii) Assume that $w \in C^{2}(\Omega) \cap C^{1}(\bar{\Omega})$ and satisfies

$$
\begin{gathered}
\Delta w(x)+g(x, w(x)) \leqslant 0 \quad \text { in } \Omega, \partial_{\nu} w \geqslant 0 \quad \text { on } \partial \Omega . \\
\text { If } w\left(x_{0}\right)=\min _{\bar{\Omega}} w \text {, then } g\left(x_{0}, w\left(x_{0}\right)\right) \leqslant 0 .
\end{gathered}
$$

Lemma 2 [Harnack Inequality [14]]. Assume that $c \in C(\bar{\Omega})$ and let $w \in$ $C^{2}(\Omega) \cap C^{1}(\bar{\Omega})$ be a positive solution to

$$
\Delta w(x)+c(x) w(x)=0 \quad \text { in } \Omega, \partial_{\nu} w=0 \quad \text { on } \partial \Omega .
$$

Then there exists a positive constant $C^{*}=C^{*}\left(\|c\|_{\infty}, \Omega\right)$ such that

$$
\max _{\bar{\Omega}} w \leq C^{*} \min _{\bar{\Omega}} w .
$$

Note that the positive solution $(u, v)$ satisfying (1.3) refers to a classical one, so we can apply the above two lemmas. For convenience, we denote the constants $r, \beta, m$ collected by $\Lambda$.

Theorem 1. Assume that $\beta \leq r m$. Let $D_{1}, D_{2}$ be arbitrary fixed positive constants. Then there exist positive constants $C_{1}=C_{1}\left(\Lambda, D_{1}, D_{2}\right)$ and $C_{2}=$ $C_{2}\left(\Lambda, D_{1}, D_{2}\right)$ such that any positive solution $(u, v)$ of (1.3) satisfies

$$
C_{2}<u(x), v(x)<C_{1}, \quad \forall x \in \bar{\Omega},
$$

provided that $d_{1}, d_{2} \geq D_{1}, d_{3} / d_{2} \leq D_{2}$. 
Proof. Since

$$
-d_{1} \Delta u=r u(1-u)-\frac{\beta u v}{u+m v} \leq r u(1-u),
$$

we can easily get $\max _{\bar{\Omega}} u \leq 1$ by the maximum principle.

Let $\varphi(x)=v\left(1+d_{3} u\right)$ and assume $\varphi\left(x_{0}\right)=\max _{\bar{\Omega}} \varphi(x)$. Then, by the maximum principle, we have $v\left(x_{0}\right) \leq u\left(x_{0}\right) \leq 1$. Therefore,

$$
\begin{aligned}
\max _{\bar{\Omega}} v(x) & =\max _{\bar{\Omega}} \frac{\varphi(x)}{1+d_{3} u} \\
& \leq \max _{\bar{\Omega}} \varphi(x)=v\left(x_{0}\right)\left(1+d_{3} u\left(x_{0}\right)\right) \leq 1+d_{3} .
\end{aligned}
$$

In the following, we prove that $(u, v)$ has a positive lower bound. Let

$$
c_{1}(x)=\frac{1}{d_{1}}\left[r(1-u)-\frac{\beta v}{u+m v}\right], \quad c_{2}(x)=\frac{1}{d_{2}} \frac{1-v / u}{1+d_{3} u} .
$$

Thus, $u$ and $\varphi$ satisfy

$$
\begin{cases}\Delta u+c_{1}(x) u=0 & \text { in } \Omega, \partial_{\nu} u=0 \quad \text { on } \partial \Omega \\ \Delta \varphi+c_{2}(x) \varphi=0 & \text { in } \Omega, \partial_{\nu} \varphi=0 \quad \text { on } \partial \Omega .\end{cases}
$$

We can find positive constants $\tilde{C}\left(D_{1}, D_{2}, \Lambda\right)$ and $C^{*}=C^{*}\left(D_{1}, D_{2}, \Lambda\right)$ such that

$$
\left\|c_{1}\right\|_{\infty}=\left\|\frac{1}{d_{1}}\left[r(1-u)-\frac{\beta v}{u+m v}\right]\right\|_{\infty} \leq \tilde{C} .
$$

Then, we have that $\max _{\bar{\Omega}} u \leq C^{*} \min _{\bar{\Omega}} u$ by the Harnack inequality. Furthermore,

$$
\begin{aligned}
\left\|c_{2}\right\|_{\infty} & =\left\|\frac{1}{d_{2}} \frac{1-v / u}{1+d_{3} u}\right\|_{\infty}=\left\|\frac{1}{d_{2}} \frac{u-v}{\left(1+d_{3} u\right) u}\right\|_{\infty} \leq \frac{1}{d_{2}}+\frac{\max _{\bar{\Omega}} v}{d_{2} \min _{\bar{\Omega}} u} \\
& \leq C+\frac{\max _{\bar{\Omega}} \varphi}{d_{2} \min _{\bar{\Omega}} u}=C+\frac{v\left(x_{0}\right)\left(1+d_{3} u\left(x_{0}\right)\right)}{d_{2} \min _{\bar{\Omega}} u} \\
& \leq C+\frac{u\left(x_{0}\right)\left(1+d_{3}\right)}{d_{2} \min _{\bar{\Omega}} u} \leq C+\frac{\left(1+d_{3}\right)}{d_{2}} \frac{\max _{\bar{\Omega}} u}{\min _{\bar{\Omega}} u} \leq \tilde{C} .
\end{aligned}
$$

So, we get that $\max _{\bar{\Omega}} \varphi \leq C^{*} \min _{\bar{\Omega}} \varphi$.

Then, there is a constant $C_{1}^{*}=C_{1}^{*}\left(D_{1}, D_{2}, \Lambda\right)$ such that

$$
\frac{\max _{\bar{\Omega}} v}{\min _{\bar{\Omega}} v} \leqslant \frac{\max _{\bar{\Omega}} \varphi}{\min _{\bar{\Omega}} \varphi} \cdot \frac{\max _{\bar{\Omega}}\left(1+d_{3} u\right)}{\min _{\bar{\Omega}}\left(1+d_{3} u\right)} \leqslant C^{*} \frac{\max _{\bar{\Omega}} u}{\min _{\bar{\Omega}} u} \leqslant C_{1}^{*} .
$$

By way of contradiction, we suppose that $(u, v)$ does not have a positive lower bound, then there is a sequence $\left\{d_{1, i}, d_{2, i}, d_{3, i}\right\}_{i=1}^{\infty}, d_{1, i}, d_{2, i} \geq D_{1}, d_{3, i} \geq$ 0 , such that the positive solution $\left(u_{i}, v_{i}\right)$ of $(1.3)$ corresponding to $\left(d_{1}, d_{2}, d_{3}\right)=$ $\left(d_{1, i}, d_{2, i}, d_{3, i}\right)$ satisfies

$$
\min _{\bar{\Omega}} u_{i}(x) \rightarrow 0 \quad \text { or } \quad \min _{\bar{\Omega}} v_{i}(x) \rightarrow 0 \quad \text { as } \quad i \rightarrow \infty,
$$


where $\left(u_{i}, v_{i}\right)$ solves the following equations

$$
\left\{\begin{array}{l}
-d_{1, i} \Delta u_{i}=r u_{i}\left(1-u_{i}\right)-\frac{\beta u_{i} v_{i}}{u_{i}+m v_{i}} \quad \text { in } \Omega, \\
-d_{2, i} \Delta\left[\left(1+d_{3, i} u_{i}\right) v_{i}\right]=v_{i}\left(1-v_{i} / u_{i}\right) \quad \text { in } \Omega, \\
\partial_{\nu} u_{i}=\partial_{\nu} v_{i}=0 \quad \text { on } \partial \Omega .
\end{array}\right.
$$

Integrating over $\Omega$ by parts for (2.1) yields

$$
\int_{\Omega} u_{i}\left[r\left(1-u_{i}\right)-\frac{\beta v_{i}}{u_{i}+m v_{i}}\right] \mathrm{d} x=0, \quad \int_{\Omega} v_{i}\left(1-\frac{v_{i}}{u_{i}}\right) \mathrm{d} x=0 .
$$

Then there exists $x_{i} \in \bar{\Omega}$ such that $v_{i}\left(x_{i}\right)=u_{i}\left(x_{i}\right)$ for all $i \geq 1$. So, based on the above argument, we deduce that both $u_{i}$ and $v_{i}$ converge to zero uniformly on $\bar{\Omega}$ as $i \rightarrow \infty$. Note that $\varphi=\left(1+d_{3} u\right) v$, we can rewrite (1.3) as

$$
\left\{\begin{array}{l}
-d_{1} \Delta u=u\left[r(1-u)-\frac{\beta \varphi}{u\left(1+d_{3} u\right)+m \varphi}\right] \quad \text { in } \Omega \\
-d_{2} \Delta \varphi=\frac{\varphi}{1+d_{3} u}\left(1-\frac{\varphi}{u\left(1+d_{3} u\right)}\right) \quad \text { in } \Omega \\
\partial_{\nu} u_{i}=\partial_{\nu} v_{i}=0 \quad \text { on } \partial \Omega
\end{array}\right.
$$

then it is clear that $u_{i} \rightarrow 0, \varphi_{i} \rightarrow 0$ as $i \rightarrow \infty$. Let

$$
w_{i}=\frac{u_{i}}{\left\|u_{i}\right\|_{\infty}+\left\|\varphi_{i}\right\|_{\infty}} \text { and } z_{i}=\frac{\varphi_{i}}{\left\|u_{i}\right\|_{\infty}+\left\|\varphi_{i}\right\|_{\infty}} .
$$

Then $\left(w_{i}, z_{i}\right)$ satisfies

$$
\left\{\begin{array}{l}
-d_{1, i} \Delta w_{i}=w_{i}\left[r\left(1-u_{i}\right)-\frac{\beta z_{i}}{w_{i}\left(1+d_{3, i} u_{i}\right)+m z_{i}}\right] \quad \text { in } \Omega, \\
-d_{2, i} \Delta z_{i}=\frac{z_{i}}{1+d_{3, i} u_{i}}\left(1-\frac{z_{i}}{w_{i}\left(1+d_{3, i} u_{i}\right)}\right) \quad \text { in } \Omega \\
\partial_{\nu} w_{i}=\partial_{\nu} z_{i}=0 \quad \text { on } \partial \Omega .
\end{array}\right.
$$

Integrating over $\Omega$, we have

$$
\left\{\begin{array}{l}
\int_{\Omega} w_{i}\left[r\left(1-u_{i}\right)-\frac{\beta z_{i}}{w_{i}\left(1+d_{3, i} u_{i}\right)+m z_{i}}\right] \mathrm{d} x=0 \\
\int_{\Omega} \frac{z_{i}}{1+d_{3, i} u_{i}}\left[1-\frac{z_{i}}{w_{i}\left(1+d_{3, i} u_{i}\right)}\right] \mathrm{d} x=0 .
\end{array}\right.
$$

Since $d_{1, i}, d_{2, i} \geq D_{1}$, by the standard regularity theorem for elliptic equations, we can get the existence of a subsequence $\left(w_{i}, z_{i}\right) \rightarrow(w, z)$ for some nonnegative functions $w, z$. Since $\left\|w_{i}\right\|_{\infty}+\left\|z_{i}\right\|_{\infty}=1$, we obtain

$$
\|w\|_{\infty}+\|z\|_{\infty}=1 \text {. }
$$

By the expression of $\left(w_{i}, z_{i}\right)$, we know that $\left(w_{i}, z_{i}\right)$ satisfies the Harnack inequality. Thus, by taking $i \rightarrow \infty$, we can conclude that $(w, z)$ satisfies the Harnack inequality. Hence, if $\omega(x)$ or $z(x)$ is equal to zero at some point $x_{0}$, then 
$w(x) \equiv 0$ or $z(x) \equiv 0, x \in \bar{\Omega}$ by the Harnack inequality. As $\|w\|_{\infty}+\|z\|_{\infty}=1$, consequently, if $\omega\left(x_{0}\right)=0$ for some point $x_{0}$, then $w(x) \equiv 0,\|w\|_{\infty}=0$, thus $z(x)$ can not attain zero at any point $x \in \bar{\Omega}, z(x)>0, x \in \bar{\Omega}$. So $w(x)+z(x)>0$ on $\bar{\Omega}$. If $z\left(x_{0}\right)=0$ for some point $x_{0} \in \Omega$, similarly, we can obtain $w(x)+z(x)>0$ on $\bar{\Omega}$. Therefore, we have $w(x)+z(x)>0$ on $\bar{\Omega}$ in any case. Letting $i \rightarrow \infty$ in (2.5), we get

$$
\int_{\Omega} w\left[r-\frac{\beta z}{w+m z}\right] \mathrm{d} x=0, \quad \int_{\Omega} z\left[1-\frac{z}{w}\right] \mathrm{d} x=0 .
$$

By a simple observation, we have neither $w \equiv 0$ nor $z \equiv 0$, thus $w>0, z>0$. While

$$
\int_{\Omega} w\left[r-\frac{\beta z}{w+m z}\right] \mathrm{d} x=\int_{\Omega} w \frac{r w+(r m-\beta) z}{w+m z} \mathrm{~d} x=0,
$$

and $\beta \leq r m$, we get a contradiction. This completes the proof.

\section{Non-existence of Non-constant Positive Solutions}

In this section, we shall give some sufficient conditions for the non-existence of non-constant positive solutions of (1.3).

Theorem 2. Let the assumptions in Theorem 1 hold. Assume that $\varepsilon$ is an arbitrary positive constant and $D_{1}, D_{2}$ are two given positive constants. Then there exists a positive constant $C=C\left(\Lambda, D_{1}, D_{2}, \varepsilon\right)$ such that (1.3) has no non-constant positive solutions provided that $d_{1}>C\left(1+d_{2}^{2} d_{3}^{2}\right)$.

Proof. Let $(u, v)$ be a positive solution of (1.3) and denote $\bar{g}=\frac{1}{\Omega} \int_{\Omega} g \mathrm{dx}$ for any $g \in L^{1}(\Omega)$. Assume $d_{1}, d_{2} \geq D_{1}$, then multiplying the corresponding equation in (1.3) by $\frac{u-\bar{u}}{u}$ and $\frac{v-\bar{v}}{v}$, respectively, and integrating over $\Omega$, we have that

$$
\begin{aligned}
\int_{\Omega} & \left\{d_{1} \frac{\bar{u}}{u^{2}}|\nabla u|^{2}+\frac{d_{2}\left(1+d_{3} u\right) \bar{v}}{v^{2}}|\nabla v|^{2}+\frac{d_{2} d_{3} \bar{v}}{v} \nabla u \cdot \nabla v\right\} \mathrm{d} x \\
= & \int_{\Omega}\left\{\left[r(1-u)-\frac{\beta v}{u+m v}\right](u-\bar{u})+\left(1-\frac{v}{u}\right)(v-\bar{v})\right\} \mathrm{d} x \\
= & \int_{\Omega}\left\{(u-\bar{u})^{2}\left[-r+\frac{\beta \bar{v}}{(u+m v)(\bar{u}+m \bar{v})}\right]\right. \\
& \left.+\left[-\frac{\beta \bar{u}}{(u+m v)(\bar{u}+m \bar{v})}+\frac{\bar{v}}{u \bar{u}}\right](u-\bar{u})(v-\bar{v})-\frac{1}{u}(v-\bar{v})^{2}\right\} \mathrm{d} x
\end{aligned}
$$

then, by Theorem 1 and the $\varepsilon$-Young Inequality, we get

$$
\begin{aligned}
& \int_{\Omega}\left\{d_{1} \frac{\bar{u}}{u^{2}}|\nabla u|^{2}+\frac{d_{2}\left(1+d_{3} u\right) \bar{v}}{v^{2}}|\nabla v|^{2}\right\} \mathrm{d} x \\
& \quad \leq \int_{\Omega}\left\{C_{1}(\varepsilon)(u-\bar{u})^{2}+\left(\varepsilon-\frac{1}{u}\right)(v-\bar{v})^{2}+\frac{d_{2}^{2} d_{3}^{2} \bar{v}^{2}}{4 \varepsilon v^{2}}|\nabla u|^{2}+\varepsilon|\nabla v|^{2}\right\} \mathrm{d} x,
\end{aligned}
$$


where $\varepsilon$ is an arbitrary small positive constant arising from Young's inequality, $C_{1}(\varepsilon)$ depends only on $\Lambda, \Omega, \varepsilon$. It follows from the Poincaré inequality that

$$
\int_{\Omega}\left\{d_{1} \frac{\bar{u}}{u^{2}}|\nabla u|^{2}+\frac{d_{2}\left(1+d_{3} u\right) \bar{v}}{v^{2}}|\nabla v|^{2}\right\} \mathrm{d} x \leq \int_{\Omega} C(\varepsilon)\left(1+d_{2}^{2} d_{3}^{2}\right)|\nabla u|^{2} \mathrm{~d} x .
$$

Hence, we can deduce that there exists a positive constant $C=C\left(\Lambda, D_{1}, D_{2}, \varepsilon\right)$ such that $(u, v) \equiv(\bar{u}, \bar{v})$ if $d_{1}>C\left(1+d_{2}^{2} d_{3}^{2}\right)$, which asserts our result.

Let $\lambda_{1}$ be the smallest positive eigenvalue of the operator $-\Delta$ under homogeneous Neumann boundary condition over $\Omega$.

Theorem 3. Let the assumptions in Theorem 1 hold. Assume that $\varepsilon$ is an arbitrary positive constant and $D_{1}, D_{2}$ are two given positive constants. Then there exists a positive constant $C=C\left(\Lambda, D_{1}, D_{2}, \varepsilon\right)$ such that (1.3) has no positive non-constant solutions provided that

$$
d_{1}>r / \lambda_{1}+\varepsilon, d_{2}>C\left(1+d_{2}^{2} d_{3}^{2}\right) .
$$

Proof. Let $(u, v)$ be a positive solution of (1.3) and $\bar{g}=\frac{1}{|\Omega|} \int_{\Omega} g \mathrm{dx}$ for any $g \in L^{1}(\Omega)$. Multiplying the first equation of $(1.3)$ by $(u-\bar{u})$, we have

$$
\begin{aligned}
\int_{\Omega} & d_{1}|\nabla u|^{2} \mathrm{~d} x=\int_{\Omega}\left[r u(1-u)-\frac{\beta u v}{u+m v}\right](u-\bar{u}) \mathrm{d} x \\
= & \int_{\Omega}\left\{(u-\bar{u})^{2}\left[r-r(u+\bar{u})-\frac{\beta m v \bar{v}}{(u+m v)(\bar{u}+m \bar{v})}\right]\right. \\
& \left.-\frac{\beta u \bar{u}}{(u+m v)(\bar{u}+m \bar{v})}(u-\bar{u})(v-\bar{v})\right\} \mathrm{d} x \\
\leq & \int_{\Omega}\left\{\left(r+\varepsilon_{1}\right)(u-\bar{u})^{2}+C_{1}\left(\varepsilon_{1}\right)(v-\bar{v})^{2}\right\} \mathrm{dx}
\end{aligned}
$$

where $\varepsilon_{1}$ is an arbitrary small positive constant arising from Young's inequality, $C_{1}\left(\varepsilon_{1}\right)$ depends only on $\Lambda, \Omega, \varepsilon$.

Multiplying the second equation of $(1.3)$ by $(v-\bar{v})$, we get

$$
\begin{gathered}
\int_{\Omega}\left\{d_{2}\left(1+d_{3} u\right)|\nabla v|^{2}+d_{2} d_{3} v \nabla u \cdot \nabla v\right\} \mathrm{d} x=\int_{\Omega}\left(v-\frac{v^{2}}{u}\right)(v-\bar{v}) \mathrm{d} x \\
=\int_{\Omega}\left\{\left(1-\frac{v+\bar{v}}{u}\right)(v-\bar{v})^{2}+\frac{\bar{v}^{2}}{u \bar{u}}(u-\bar{u})(v-\bar{v})\right\} \mathrm{d} x .
\end{gathered}
$$

Thus, following the Poincaré inequality, we have that

$$
\begin{aligned}
& \int_{\Omega} d_{2}\left(1+d_{3} u\right)|\nabla v|^{2} \mathrm{~d} x \\
& \quad=\int_{\Omega}\left\{(v-\bar{v})^{2}\left(1-\frac{v+\bar{v}}{u}\right)+\frac{\bar{v}^{2}}{u \bar{u}}(u-\bar{u})(v-\bar{v})-d_{2} d_{3} v \nabla u \cdot \nabla v\right\} \mathrm{d} x \\
& \quad \leq \int_{\Omega}\left\{\varepsilon_{1}(u-\bar{u})^{2}+C_{2}\left(\varepsilon_{1}\right)(v-\bar{v})^{2}+\varepsilon_{1}|\nabla u|^{2}+\frac{d_{2}^{2} d_{3}^{2} v^{2}}{4 \varepsilon_{1}}|\nabla v|^{2}\right\} \mathrm{d} x \\
& \quad \leq \int_{\Omega}\left\{\left(1+1 / \lambda_{1}\right) \varepsilon_{1}|\nabla u|^{2}+C_{3}\left(\varepsilon_{1}\right)\left(1+d_{2}^{2} d_{3}^{2}\right)|\nabla v|^{2}\right\} \mathrm{d} x
\end{aligned}
$$


where $C_{2}\left(\varepsilon_{1}\right)$ and $C_{3}\left(\varepsilon_{1}\right)$ depend on $\Lambda, \Omega, \varepsilon_{1}$. Then,

$$
\begin{aligned}
& \int_{\Omega} d_{1}|\nabla u|^{2}+d_{2}\left(1+d_{3} u\right)|\nabla v|^{2} \mathrm{~d} x \\
& \quad \leq \int_{\Omega}\left(r / \lambda_{1}+\varepsilon\right)|\nabla u|^{2}+C(\varepsilon)\left(1+d_{2}^{2} d_{3}^{2}\right)|\nabla v|^{2} \mathrm{~d} x
\end{aligned}
$$

By the above inequality, we can see that there exists a positive constant $C=$ $C\left(\Lambda, D_{1}, D_{2}, \varepsilon\right)$ such that $(u, v) \equiv(\bar{u}, \bar{v})$ if

$$
d_{1}>r / \lambda_{1}+\varepsilon, \quad d_{2}>C(\varepsilon)\left(1+d_{2}^{2} d_{3}^{2}\right),
$$

which asserts our result. The proof is completed.

\section{Existence of Non-constant Positive Solutions}

In this section, we are concerned with the existence of the non-constant positive solutions of (1.3). In particular, by applying the Leray-Schauder topological degree theory, we show that for certain ranges of parameters, system (1.3) can produce patterns while the system without cross-diffusion does not have any non-constant positive steady state.

Let $w=v\left(1+d_{3} u\right)$ and $w^{*}=v^{*}\left(1+d_{3} u^{*}\right)$. Denote $\tilde{\boldsymbol{u}}=\left(u^{*}, w^{*}\right), \boldsymbol{u}=(u, w)$. Clearly, (1.3) is equivalent to the following equation

$$
(\boldsymbol{I}-\boldsymbol{G})(\boldsymbol{u})=0
$$

where

$$
\boldsymbol{G}(\boldsymbol{u})=\left(\begin{array}{c}
\left(\boldsymbol{I}-d_{1} \Delta\right)^{-1}\left[r u(1-u)+u-\frac{\beta u w}{u\left(1+d_{3} u\right)+m w}\right] \\
\left(\boldsymbol{I}-d_{2} \Delta\right)^{-1}\left[w+\frac{w}{1+d_{3} u}\left(1-\frac{w}{u\left(1+d_{3} u\right)}\right)\right]
\end{array}\right),
$$

$\left(\boldsymbol{I}-d_{i} \Delta\right)^{-1}(i=1,2)$ is the inverse of $\boldsymbol{I}-d_{i} \Delta$ with homogeneous Neumann boundary condition. As the operator $\left(\boldsymbol{I}-d_{i} \Delta\right)^{-1}: C(\bar{\Omega}) \rightarrow C(\bar{\Omega})(i=1,2)$ exist and are compact, $\boldsymbol{G}:[C(\bar{\Omega})]^{2} \rightarrow[C(\bar{\Omega})]^{2}$ is also compact. To apply the degree theory, we first compute the index of $\boldsymbol{I}-\boldsymbol{G}$ at $\tilde{\boldsymbol{u}}$. Consider the following eigenvalue problem

$$
-(\boldsymbol{I}-D \boldsymbol{u} \boldsymbol{G}(\tilde{\boldsymbol{u}}))(y, z)=\mu(y, z), \quad(y, z) \neq(0,0) .
$$

By the Leray-Schauder theorem (see [18, P37-38]), we have that if 0 is not the eigenvalue of (4.2), then

$$
\operatorname{index}(\boldsymbol{I}-\boldsymbol{G}, \tilde{\boldsymbol{u}})=(-1)^{\ell}, \quad \ell=\sum_{\mu>0} n_{\mu},
$$

where $n_{\mu}$ is the multiplicity of the positive eigenvalue $\mu$ of (4.2). After some computations, we can get that finding the solutions of (4.2) is equivalent to 
solving

$$
\left\{\begin{array}{c}
-d_{1}(\mu+1) \Delta y+\left[\mu-r+2 r u^{*}+\frac{\beta\left(m+(m-1) d_{3} u^{*}\right)}{(m+1)^{2}\left(1+d_{3} u^{*}\right)}\right] y \\
+\frac{\beta}{(m+1)^{2}\left(1+d_{3} u^{*}\right)} z=0 \\
-d_{2}(\mu+1) \Delta z-\frac{1+2 d_{3} u^{*}}{1+d_{3} u^{*}} y+\left(\mu+\frac{1}{1+d_{3} u^{*}}\right) z=0 \quad \text { in } \Omega \\
\partial_{\nu} y=\partial_{\nu} z=0 \quad \text { on } \partial \Omega .
\end{array}\right.
$$

Let $0=\lambda_{0}<\lambda_{1}<\cdots<\lambda_{k}<\cdots$ denote the eigenvalues of the operator $-\Delta$ subject to the homogeneous Neumann boundary condition. From the above, we see that $\mu$ is an eigenvalue of (4.2) if and only if $P_{k}(\mu)=0$ for some $k \geq 0$, where

$$
P_{k}(\mu)=\operatorname{det}\left(\begin{array}{cc}
\mu+\frac{d_{1} \lambda_{k}-r+2 r u^{*}+\frac{\beta\left(m+(m-1) d_{3} u^{*}\right)}{(m+1)^{2}\left(1+d_{3} u^{*}\right)}}{1+d_{1} \lambda_{k}} & \frac{\beta}{(1+m)^{2}\left(1+d_{3} u^{*}\right)\left(1+d_{1} \lambda_{k}\right)} \\
-\frac{1+2 d_{3} u^{*}}{\left(1+d_{2} \lambda_{k}\right)\left(1+d_{3} u^{*}\right)} & \mu+\frac{d_{2} \lambda_{k}+1 /\left(1+d_{3} u^{*}\right)}{1+d_{2} \lambda_{k}}
\end{array}\right) .
$$

Let $m_{\mu}$ denote the multiplicity of $\mu$ for the positive solution of $P_{k}(\mu)=0$ and $\ell_{k}$ denote the multiplicity of $\lambda_{k}$. Then, by (4.3), we get that if $P_{k}(0) \neq 0$ for all $k \geq 0$,

$$
\operatorname{index}(\boldsymbol{I}-\boldsymbol{G}, \tilde{\boldsymbol{u}})=(-1)^{\ell}, \quad \ell=\sum_{k=0}^{\infty} \sum_{\mu>0, P_{k}(\mu)=0} m_{\mu} \ell_{k} .
$$

Lemma 3. If $d_{3}=0$, then there is a positive constant $\bar{D}_{1}=\bar{D}_{1}\left(d_{2}, \Lambda\right)$ such that index $(\boldsymbol{I}-\boldsymbol{G}, \tilde{\boldsymbol{u}})=1$ for all $d_{1} \geq \bar{D}_{1}$.

Proof. If $d_{3}=0$, then system (1.3) reduces to

$$
\left\{\begin{array}{l}
-d_{1} \Delta u=r u(1-u)-\frac{\beta u v}{u+m v}, \quad \text { in } \Omega \\
-d_{2} \Delta v=v(1-v / u), \quad \text { in } \Omega \\
\partial_{\nu} u=\partial_{\nu} v=0, \quad \text { on } \partial \Omega .
\end{array}\right.
$$

Thus, the corresponding $P_{k}(\mu)$ is

$$
\begin{aligned}
P_{k}(\mu) & =\operatorname{det}\left(\begin{array}{cc}
\mu+\frac{d_{1} \lambda_{k}-r+2 r u^{*}+\beta m /(m+1)^{2}}{1+d_{1} \lambda_{k}} & \frac{\beta}{(1+m)^{2}\left(1+d_{1} \lambda_{k}\right)} \\
-\frac{1}{1+d_{2} \lambda_{k}} & \mu+1
\end{array}\right) \\
& =\operatorname{det}\left(\begin{array}{cc}
\mu+\frac{d_{1} \lambda_{k}+(r / m+1)\left((m+2) u^{*}-1\right)}{1+d_{1} \lambda_{k}} & \frac{\beta}{(1+m)^{2}\left(1+d_{1} \lambda_{k}\right)} \\
-\frac{1}{1+d_{2} \lambda_{k}} & \mu+1
\end{array}\right) .
\end{aligned}
$$

For $k=0, \lambda_{0}=0$ and the constant term of $P_{0}(\mu)$ is

$$
\frac{r}{m+1}\left[(m+2) u^{*}-1\right]+\frac{\beta}{(m+1)^{2}}=r u^{*}>0,
$$


thus $\mu=0$ is not the root of $P_{0}(\mu)=0$. For $k \geq 1$, we have $\lambda_{k} \geq \lambda_{1}>0$ and $\lim _{d_{1} \rightarrow \infty} P_{k}(\mu)=(\mu+1)^{2}$ for all $k \geq 1$, then there exists a constant $\bar{D}_{1}$ such that for all $k \geq 1, P_{k}(\mu)$ has no roots with positive real parts provided $d_{1} \geq \bar{D}_{1}$. Consequently, from (4.5), we get

$$
\operatorname{index}(\boldsymbol{I}-\boldsymbol{G}, \tilde{\boldsymbol{u}})=(-1)^{r_{0}}, \quad r_{0}=\sum_{\mu>0, P_{0}(\mu)=0} m_{\mu} .
$$

Moreover, since the constant term of $P_{0}(\mu)$ is positive, we obtain index $(\boldsymbol{I}-$ $\boldsymbol{G}, \tilde{\boldsymbol{u}})=1$, which asserts our result.

For all $k \geq 0$, by some computations, we obtain that the constant term of $P_{k}(\mu)$ is

$$
M(k)=\frac{H\left(d_{3}\right)}{\left(1+d_{1} \lambda_{k}\right)\left(1+d_{2} \lambda_{k}\right)\left(1+d_{3} u^{*}\right)},
$$

where $H\left(d_{3}\right)$ is defined as

$$
\begin{aligned}
H\left(d_{3}\right)= & d_{3} u^{*}\left[d_{1} d_{2} \lambda_{k}^{2}+d_{2} \lambda_{k}\left(r\left(2 u^{*}-1\right)+\frac{\beta(m-1)}{(m+1)^{2}}\right)\right]+r u^{*}+d_{1} \lambda_{k} \\
& +d_{1} d_{2} \lambda_{k}^{2}+d_{2} \lambda_{k}\left[r\left(2 u^{*}-1\right)+\beta m /(m+1)^{2}\right] .
\end{aligned}
$$

Let $Q=r\left(1-2 u^{*}\right)-\beta(m-1) /(m+1)^{2}$. We assume that $Q>0$, and $H\left(d_{3}\right) \neq 0$ for all $k \geq 0$.

Remark 1. Since

$$
Q=r\left(1-2 u^{*}\right)-\frac{\beta(m-1)}{(m+1)^{2}}=\frac{\beta(m+3)-r(m+1)^{2}}{(m+1)^{2}}
$$

then $Q>0 \Leftrightarrow \beta>r(m+1)^{2} /(m+3)$. As we assume $\beta<r m$, it is necessary to assume that

$$
r m>r(m+1)^{2} /(m+3) .
$$

This condition is equivalent to $m>1$, and thus we need the condition $m>1$.

We can see that

$$
P_{k}=\left(\mu+\frac{d_{2} \lambda_{k}}{1+d_{2} \lambda_{k}}\right)\left(\mu+\frac{d_{1} \lambda_{k}-Q}{1+d_{1} \lambda_{k}}\right)+\mathcal{O}\left(\frac{1}{d_{3}}\right) .
$$

Consequently, we assume that $Q / d_{1} \in\left(\lambda_{k^{*}}, \lambda_{k^{*}+1}\right)$, as equation

$$
\left(\mu+\frac{d_{2} \lambda_{k}}{1+d_{2} \lambda_{k}}\right)\left(\mu+\frac{d_{1} \lambda_{k}-Q}{1+d_{1} \lambda_{k}}\right)=0
$$

has only one positive root for $k \leq k^{*}$ and the roots are all negative for $k>k^{*}$. Thus, we can find a positive constant $\widetilde{D}$ such that the polynomial $P_{k}(\mu)=0$ has only one simple positive root for $k \leq k^{*}$ while all roots of $P_{k}(\mu)=0$ have negative real parts for $k>k^{*}$ when $d_{3} \geq \widetilde{D}$. So we obtain the following lemma. 
Lemma 4. Assume that $H\left(d_{3}\right) \neq 0$ for all $k \geq 0, Q>0$ and $\frac{Q}{d_{1}} \in\left(\lambda_{k^{*}}, \lambda_{k^{*}+1}\right)$ for some $k^{*} \geq 0$. Then there exists a positive constant $\widetilde{D}=\widetilde{D}\left(D_{1}, D_{2}, \Lambda\right)$ such that for all $d_{3} \geq \widetilde{D}$,

$$
\operatorname{index}(\boldsymbol{I}-\boldsymbol{G}, \tilde{\boldsymbol{u}})=(-1)^{\ell}, \quad \ell=\sum_{i=0}^{k^{*}} \ell_{i} .
$$

Theorem 4. Suppose that the assumptions in Theorem 1 and Lemma 4 hold. If $\sum_{i=0}^{k^{*}} \ell_{i}$ is odd, then there exists a positive constant $\bar{D}=\bar{D}\left(D_{1}, D_{2}, \Lambda\right)$ such that for all $d_{3} \geq \bar{D},(1.3)$ has at least one non-constant positive solution.

Proof. To prove the theorem, we assume (1.3) has no non-constant positive solution for some $d_{3}^{*} \geq \bar{D}$. Fix $\bar{d}_{2}>D_{1}$ and let $\bar{d}_{1}$ be large enough such that Theorem 2 and Lemma 3 hold for $d_{3}=0$. For $0 \leqslant t \leqslant 1$, we define

$$
\boldsymbol{G}(\boldsymbol{u}, t)=\left(\begin{array}{c}
\left(\boldsymbol{I}-\left[t d_{1}+(1-t) \bar{d}_{1}\right] \Delta\right)^{-1}\left[r u(1-u)+u-\frac{\beta u w}{u\left(1+t d_{3}^{*} u\right)+m w}\right] \\
\left(\boldsymbol{I}-\left[t d_{2}+(1-t) \bar{d}_{2}\right] \Delta\right)^{-1}\left[w+\frac{w}{1+t d_{3}^{*} u}\left(1-\frac{w}{u\left(1+t d_{3}^{*} u\right)}\right)\right]
\end{array}\right)
$$

By Theorem 1, there exists a positive constant $C_{1}$ such that (4.1) has no positive solution on $\partial \Sigma$, where

$$
\Sigma=\left\{(u, w) \in[C(\bar{\Omega})]^{2}: 1 / C_{1}<u, w<C_{1}\right\} .
$$

Since $\boldsymbol{G}(\boldsymbol{u}, t): \Sigma \times[0,1] \rightarrow[C(\bar{\Omega})]^{2}$ is compact, we can define the degree $\operatorname{deg}(\boldsymbol{I}-\boldsymbol{G}(\cdot, t), \Sigma, 0)$. By the homotopy invariance of degree,

$$
\operatorname{deg}(\boldsymbol{I}-\boldsymbol{G}(\cdot, 0), \Sigma, 0)=\operatorname{deg}(\boldsymbol{I}-\boldsymbol{G}(\cdot, 1), \Sigma, 0) .
$$

Since we assume that for some $d_{3}^{*} \geqslant \widetilde{D}$, (4.1) has no non-constant positive solution, then, by Lemma 4, we have

$$
\operatorname{deg}(\boldsymbol{I}-\boldsymbol{G}(\cdot, 1), \Sigma, 0)=\operatorname{index}(\boldsymbol{G}(\cdot, 1), \tilde{\boldsymbol{u}})=(-1)^{\ell}=-1,
$$

while

$$
\operatorname{deg}(\boldsymbol{I}-\boldsymbol{G}(\cdot, 0), \Sigma, 0)=\operatorname{index}(\boldsymbol{G}(\cdot, 0), \tilde{\boldsymbol{u}})=1 .
$$

Thus, we get a contradiction. The proof is completed.

Remark 2. In order to keep the system considered in [23] in accord with system (1.3) in our paper, we take $\lambda=r=1$. In this case, by [23, Theorem 2.1], we know that the unique constant positive steady state $\boldsymbol{u}^{*}=\left(u^{*}, v^{*}\right)$ is globally asymptotically stable if

$$
\beta<\min \left\{m,(1+m)^{2} K\right\} \text { and }\left(1+u^{*}\right) \beta<(1+m)^{2} K\left(K+u^{*}\right),
$$

where $K=\frac{1}{2}\left\{(1-m)+\sqrt{(1-m)^{2}+4(m-\beta)}\right\}$. It is clear that $K<1$ and $K \rightarrow 1$ as $m \rightarrow \infty$ by some simple computations. Thus when $m$ is large enough such that $K$ sufficiently approaches to 1, (4.6) automatically holds if $\beta<m$. 
So when $m$ is large enough and $\beta<m$, the unique constant positive steady states $\boldsymbol{u}^{*}=\left(u^{*}, v^{*}\right)$ is globally asymptotically stable for system (1.3) without cross-diffusion.

Now, we suppose that $m$ is large enough and $\beta<m$. By Theorem 4 , system (1.3) has at least one non-constant positive solution if some conditions are satisfied. $Q>0$ is required in Theorem 4 , and this is equivalent to $\beta>$ $\frac{(m+1)^{2}}{m+3}$ (see Remark 1). As $\beta<m, \frac{(m+1)^{2}}{m+3}$ must be smaller than $m$. This is true when $m>1$. So we can guarantee $Q>0$.

Furthermore, if $d_{1}$ is large enough such that $\frac{Q}{d_{1}} \in\left(\lambda_{0}, \lambda_{1}\right)$, then $\sum_{i=0}^{k^{*}} \ell_{i}=$ $\ell_{0}=1$, which is odd, so we know if $H\left(d_{3}\right) \neq 0$ and the cross-diffusion $d_{3}$ is large enough, system (1.3) has at least one non-constant positive steady states.

Thus, our result shows that even though the unique constant positive steady state is globally asymptotically stable for system (1.3) without cross-diffusion, non-constant positive steady state can still exist due to the emergence of the cross-diffusion for system (1.3), which suggests that Turing pattern occurs.

\section{References}

[1] R. Arditi and L.R. Ginzburg. Coupling in predator-prey dynamics: ratiodependence. J. Theor. Biol., 139:311-326, 1989.

Doi:10.1016/S0022-5193(89)80211-5.

[2] A.A. Berryman. The origins and evolution of predator-prey theory. Ecology, 73:1530-1535, 1992. Doi:10.2307/1940005.

[3] W.Y. Chen and M.X. Wang. Qualitative analysis of predator-prey models with Beddington-DeAngelis functional response and diffusion. Math. Computer Modelling, 42:31-44, 2005. Doi:10.1016/j.mcm.2005.05.013.

[4] X.F. Chen, Y.W. Qi and M.X. Wang. A strongly coupled predator-prey system with non-monotonic functional response. Nonlinear Anal., 67:1966-1979, 2007. Doi:10.1016/j.na.2006.08.022.

[5] F.A. Davidson and B.P. Rynne. A priori bounds and global existence of solutions of the steady-state Sel'kov model. Proc. Roy. Soc. Edinburgh Sect. A, 130:507$516,2000$.

[6] Y.H. Du and Y. Lou. Qualitative behavior of positive solutions of a predatorprey model: effect of saturation. Proc. Roy. Soc. Edinburgh Sect. A, 131:321-349, 2001. Doi:10.1017/S0308210500000895.

[7] A. Gierer and H. Meinhardt. A theory of biological pattern formation. Kybemetik, 12:30-39, 1927.

[8] D. Iron, M.J. Ward and J.C. Wei. The stability of spike solutions to the one-dimensional Gierer-Meinhardt model. Phys. D, 150(1-2):25-62, 2001. Doi:10.1016/S0167-2789(00)00206-2.

[9] Y. Kan-on. Existence and instability of Neumann layer solutions for a 3component Lotka-Volterra model with diffusion. J. Math. Anal. Appl., 243:357372, 2000. Doi:10.1006/jmaa.1999.6676.

[10] Y. Kan-on and M. Mimura. Singular perturbation approach to a 3-component reaction-diffusion system arising in population dynamics. SIAM J. Math. Anal., 29:1519-1536, 1998. Doi:10.1137/S0036141097318328. 
[11] W. Ko and K. Ryu. Qualitative analysis of a predator-prey model with Holling type II functional response incorporating a prey refuge. J. Differential Equations, 231:534-550, 2006. Doi:10.1016/j.jde.2006.08.001.

[12] W. Ko and K. Ryu. Non-constant positive steady states of a diffusive predatorprey system in homogeneous environment. J. Math. Anal. Appl., 327:539-549, 2007. Doi:10.1016/j.jmaa.2006.04.077.

[13] W. Ko and K. Ryu. On a predator-prey system with cross-diffusion representing the tendency of predators in the presence of prey species. J. Math. Anal. Appl., 341:1133-1142, 2008. Doi:10.1016/j.jmaa.2007.11.018.

[14] C.S. Lin, W.M. Ni and I. Takagi. Large amplitude stationary solutions to a chemotais systems. J. Differential Equations, 72:1-27, 1988.

Doi:10.1016/0022-0396(88)90147-7.

[15] Y. Lou, S. Martinez and W.M. Ni. On $3 \times 3$ Lotka-Volterra competition systems with cross-diffusion. Discrete Contin. Dyn. Syst., 6(1):175-190, 2000.

[16] Y. Lou and W.M. Ni. Diffusion, self-diffusion and cross-diffusion. J. Differential Equations, 131:79-131, 1996. Doi:10.1006/jdeq.1996.0157.

[17] Y. Lou and W.M. Ni. Diffusion vs cross-diffusion: an elliptic approach. $J$. Differential Equations, 154:157-190, 1999. Doi:10.1006/jdeq.1998.3559.

[18] L. Nirenberg. Topics in Nonlinear Functional Analysis. American Mathematical Society, Providence, RI, 2001.

[19] P.Y.H. Pang and M.X. Wang. Qualitative analysis of a ratio-dependent predatorprey system with diffusion. Proc. Roy. Soc. Edinburgh Sect. A, 133(4):919-942, 2003. Doi:10.1017/S0308210500002742.

[20] P.Y.H. Pang and M.X. Wang. Non-constant positive steady states of a predatorprey system with non-monotonic functional response with diffusion. Proc. Lond. Math. Soc., 88:135-157, 2004. Doi:10.1112/S0024611503014321.

[21] P.Y.H. Pang and M.X. Wang. Strategy and stationary pattern in a threespecies predator-prey model. J. Differential Equations, 200:245-273, 2004. Doi:10.1016/j.jde.2004.01.004.

[22] R. Peng and M.X. Wang. Positive steady-states of the Holling-Tanner preypredator model with diffusion. Proc. Roy. Soc. Edinburgh Sect. A, 135:149-164, 2005. Doi:10.1017/S0308210500003814.

[23] R. Peng and M.X. Wang. Qualitative analysis on a diffusive prey-predator model with ratio-dependent functional response. Sci. China Ser. A, 51:2043-2058, 2008. Doi:10.1007/s11425-008-0037-8.

[24] R. Peng, M.X. Wang and G.Y. Yang. Stationary patterns of the Holling-Tanner prey-predator model with diffusion and cross-diffusion. Appl. Math. Comput., 196:570-577, 2008. Doi:10.1016/j.amc.2007.06.019.

[25] A. Turing. The chemical basis of morphogenesis. Philos. Trans. Roc. Soc. B, 237:37-72, 1952.

[26] M.X. Wang. Non-constant positive steady states of the Sel'kov model. J. Differential Equations, 190:600-620, 2003. Doi:10.1016/S0022-0396(02)00100-6.

[27] M.X. Wang. Stationary patterns of strongly coupled prey-predator models. J. Math. Anal. Appl., 292:484-505, 2004. Doi:10.1016/j.jmaa.2003.12.027.

[28] J. Wei and M. Winter. Spikes for the Gierer-Meinhardt system in two dimensions: the strong coupling case. J. Differential Equations, 178:478-518, 2002. Doi:10.1006/jdeq.2001.4019. 